\title{
Environmental GIS and Applications
}

Hermann Klug (Ed.) 